\title{
Comparison between interactive (subjective) and traditional (numerical) inversion by Genetic Algorithms
}

\author{
F. Boschetti \\ CSIRO, Exploration and Mining, \\ 39 Fairway, Nedlands, 6009 \\ Western Australia \\ fabio@ned.dem.csiro.au
}

\author{
L. Moresi \\ CSIRO, Exploration and Mining, \\ 39 Fairway, Nedlands, 6009, \\ Western Australia \\ louis@ned.dem.csiro.au
}

\begin{abstract}
Inversion algorithms employ numerical evaluation of the mismatch between model and data to guide the search for minima in parameter spaces. In an alternative approach, the numerical evaluation of data misfit can be replaced by subjective judgement of the solution quality. This widens the class of problems that can be treated within the framework of formal inverse theory, in particular including various applications in which "structural similarity" between model and data determines the quality of the fit. In this paper we compare the performance of a traditional numerical inversion with an interactive inversion, in which a priori knowledge, experience and even personal intuition are provided by the user via subjective jugement. The comparison is performed on a geological application and shows that user expertise can partly compensate for lack of sufficient constraints in the numerical inversion.
\end{abstract}

\section{Introduction}

Inversion is an important tool in many real world problems and scientific applications. It attempts to reconstruct parameter distributions from measurements of their physical responses. This is achieved by a more or less structured search into a parameter space through the use of forward modeling.

In the early stages of some scientific applications of inversion (for example in geophysics), such searches were performed manually by a human operator making adjustments to some a priori guess of the parameter setting. The search would proceed in a trial and error fashion in order to match measured data and reconstruct a reasonable model. This is often called forward modeling. Much research in the last two decades has concentrated on automating this sort of process, with the use of sophisticated inversion procedures. Automation appears to eliminate most of the subjective judgement involved in repeated forward modeling by removing any input from the operator.

In fact, the subjectivity is not removed, it is simply hidden because the presence of a priori (purely subjective) assumptions is still crucial for the successful outcome of automatic inversion procedures - although in a much more subtle way. The inherent non-uniqueness underlying most real world inverse problem results in additional information being necessary in order to select a single solution among the ensemble of infinite distributions able to fit measured data. Such a priori information may be provided in the form of 1) a specific starting model for the inverse run, 2) a specific parameterization restricting the search to predetermined geometrical/statistical patterns, or 3) extra mathematical requirement for the solution, as maximum smoothness or sharp boundaries, often chosen more for mathematical convenience than for physical reasons. Since such a priori assumptions are often hidden deep in the inverse algorithm, the 'black box' use of such tools leaves the average user unaware of the precise nature of these assumptions - and hence unable to judge whether the assumptions themselves are suited to a particular case. It should be noted that here the term 'subjective' is referred to choices that the user has to take 'a priori', i.e., independently of the data under analysis and that affect the final result of the inverse process. The crucial point is that two users taking different 'a priori' choices would necessary end up with different results.

Nevertheless, many real world problems are poorly constrained by data (geological applications being a very good example) and the expert user's subjectivity, knowledge, experience and intuition can still play a major role in their solution. The implementation of fully automated systems for data analysis and Artificial Intelligence approaches have been implemented with full success only on a limited number of real world applications.

Recently, research in Artificial Intelligence has developed systems to support artistic creativity (Takagi 1998a,b). They have been used, for example, in graphic design and music composition. The systems take advantage of fast computation to generate a suite of images or music sequences. Then, an artist looks at the different images or listens to pieces of music and ranks them according to his/her own tastes. An inversion strategy takes such judgement into account in a formal mathematical way to generate a new set of 
images/music sequences, iteratively converging towards the artist's tastes/inspiration.

In this paper we propose the extension of such techniques to scientific inverse problems, and in particular to geophysical/geological applications in which subjective judgement is necessary either to discriminate between ambiguous solutions, or to evaluate different models in the absence of sufficient constraints. In doing so we present the first step in the development of a system for interactive inversion of geophysical/geological processes. The system offers three main useful features: 1) it allows a more systematic application of forward modelling codes, as an advance on the time-consuming trial and error approach; 2) it provides a formal role for relevant geological experience and knowledge in inversion which is often extremely difficult to translate into mathematically rigorous constraints; and 3) it may suggest valid solutions falling outside the range of original expectation, by facilitating a 'brainstorm-like' process between the geoscientist and the inversion procedure.

In particular we are interested in testing the potential of such a technique compared to traditional, numerical inversion. We compare the interactive, subjectively driven, inversion to a traditional numerical inversion for a synthetic mantle convection problem. The results shows that user experience may in some cases compensate for lack of accurate data. Such a comparison with a purely numerical inversion has, to our knowledge, never been presented before in the interactive inversion literature.

\section{Interactive Inversion}

The purpose of interactive inversion is to allow the user to direct the parameter space search according to his/her subjective judgement. In order to do so the traditional numerical measure of data mismatch is replaced by the user's evaluation. Humans find it hard to express subjective judgement with absolute values, while they generally find it much easier to compare different instances of the same process and rank them according to certain criteria. Consequently interactive inversion works by producing different possible solutions and presenting them to the user for judgement and ranking. Genetic inversion (Genetic algorithm, genetic programming, etc.) works by optimising an ensemble of solutions, unlike other inverse strategies that search the solution space following one single path. Accordingly they are an obvious choice for interactive inversion applications.

Here we describe the modifications necessary to make a Genetic Algorithm work interactively.

\subsection{Interactive Genetic Algorithm}

Formally, the modifications to a Genetic Algorithm required to make it work interactively are minimal. Once a set of chromosomes is generated, it is fed to a forward code. Then a set of outputs (in the form of either images or animations) is produced. The images (or animations) are visualized and the user ranks them according to his/her subjective judgement. The ranking is then fed in to the GA which uses it to produce the next generation of chromosomes. Notice that, since ranking is implicitly present in some selection strategies (such as Linear Normalization selection, used in this work), effectively no formal algorithmic change in the code is imposed by replacing a measure of fitness with the subjective evaluation.

From an implementation point of view, some work needs to be done in order to make the subjective ranking input 'user friendly' and spare the user from tedious file editing. A user interface needs to be built that allows the user to simultaneously view all the different solutions generated by the GA, rank them easily and proceed with the GA operations, possibly within a few mouse clicks. There is also the issue of avoiding human fatigue in examining numerous solutions for many generations, which could result in lack of attention and accuracy. These issues are dealt in the Interactive Inversion literature - we refer the reader to Kishi and Takagi, 1999. In regards to our application, the description of the specific human interface is given as we lead the reader through the experiment.

\section{Interactive inversion at work}

In this experiment we attempt to reconstruct the parameters (e.g. material properties) that produce a certain 2D geological section using animations of thermal convection in the Earth mantle.

This kind of problem has implications for deep crust-mantle studies. A crucial parameter for the understanding of deep crust-mantle heat convection is the geotherm, i.e., the (increasing) temperature profile in the earth as a function of depth. The continental geotherm determines whether magmas can be generated, and the extent to which rocks undergo metamorphism and geochemical modification. The geotherm can be measured directly only in the shallowest few km's of the crust, and indirectly at greater depth through mineralogical methods when small samples are ejected in volcanic eruptions.

This is a classical coupled heat flow problem and the forward model is, in principle, very simple to solve using a finite element fluid flow code. However, in the practical case, it is extremely difficult to know the parameters of the forward model (radiogenic heat production of deep crust and mantle, 
thermal conductivity of the lower crustal rocks, viscosity of the mantle, and the global partitioning of heat flow between oceans and continents).

These uncertainties lead to an inverse problem where the parameters to be determined include the physical properties of the crust and mantle. As already mentioned, in geology, such problems are usually tackled by repeated forward modelling and a good deal of intuition based upon simple one-dimensional scaling laws. Such intuition can usually come from a user with expertise in the field.

From the inversion point of view, this is a useful test problem for three main reasons: 1) the fit to the geotherm for any given forward problem is quantitative so that, in principle, a genuine, traditional automatic inversion can be performed; 2) the practical limitations of the data can be simulated easily in the experiment. In the Earth, only the uppermost part of the geotherm can be measured with the remaining constraints coming from assumptions based on the physical processes involved in the system. 3) The problem retains significant complexity, such that ambiguous solutions are possible and need to be eliminated by recourse to estimates of geological likelihood based on experience and physical intuition. This is hard to code, directly, in a mathematical sense because we would, here, consider such features as the shape of typical boundary layer instabilities or the ways in which the flow patterns evolve over time - highly difficult to formulate in a pixel-by-pixel comparison of animations.

There is also the possibility that the additional information available in the evolution of the animation in two dimensions will allow an expert operator to speed up the inversion, as well as discard models of little geological meaning. The extent to which these assumptions are helpful in constraining the inversion can be tested explicitly.

\subsection{Experimental setup}

The user is provided with a single image representing a 2-D geological vertical section (see Figure 1). The purpose of the experiment is to deduce the parameters (e.g. material properties) of the simulation that produce this geological section after the system has evolved for a specified time. In particular the parameters to be determined are the thicknesses and thermal diffusivities of two crustal layers, the viscosity of the underlying mantle layer, and strengths of the radiogenic heat sources in each layer. This results in an eight dimensional search space.
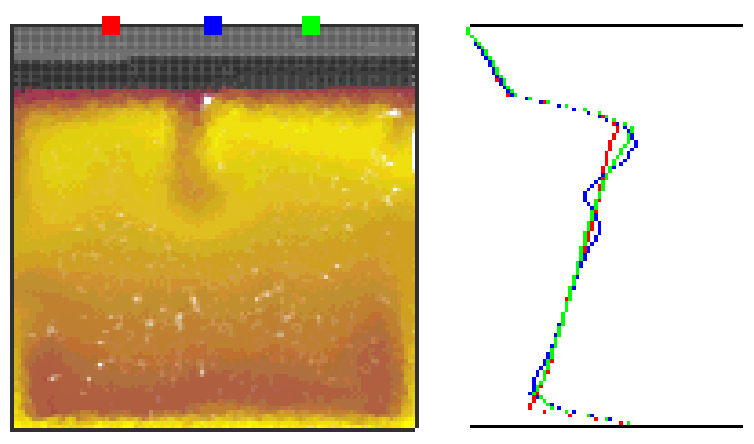

Figure 1. Target section for the synthetic test. The section has been obtained using the same forward code employed in the inversion.

At the beginning the user is presented with ten animations (see Figure 2). This number has been chosen taking into account that ranking more animations would be increasingly difficult for a human operator (notice here that normal GAs runs usually involve a much larger population). Each animation has been generated by the GA, through its standard stochastic behavior, coupled with the physical forward model. The user then views the animations and ranks them according to 1) how close the final configuration is to the target section; 2) how 'geologically feasible' the overall animation (i.e., the geological evolution) is; 3) his/her general experience and knowledge of the area under analysis. The user interface has been built in such a way that viewing of each animation is done by simple clicking of the mouse on a specific section (final frame), and the final stages of each animation are viewed together, in order to facilitate the ranking operation. The input of the ranking itself is done by a simple mouse click in small windows underneath each animation. These may appear as minor details, but are quite important in practical applications, to prevent fatigue and keep the attention of the user on the problem by sparing him/her tedious manual operations. After the ranking is done, the GA starts its usual process and generates a new set of animations for the next evaluation. The process keeps on until the user is satisfied with the result, i.e. with an animation that looks geologically reasonable and produces a final result close to the target image. 


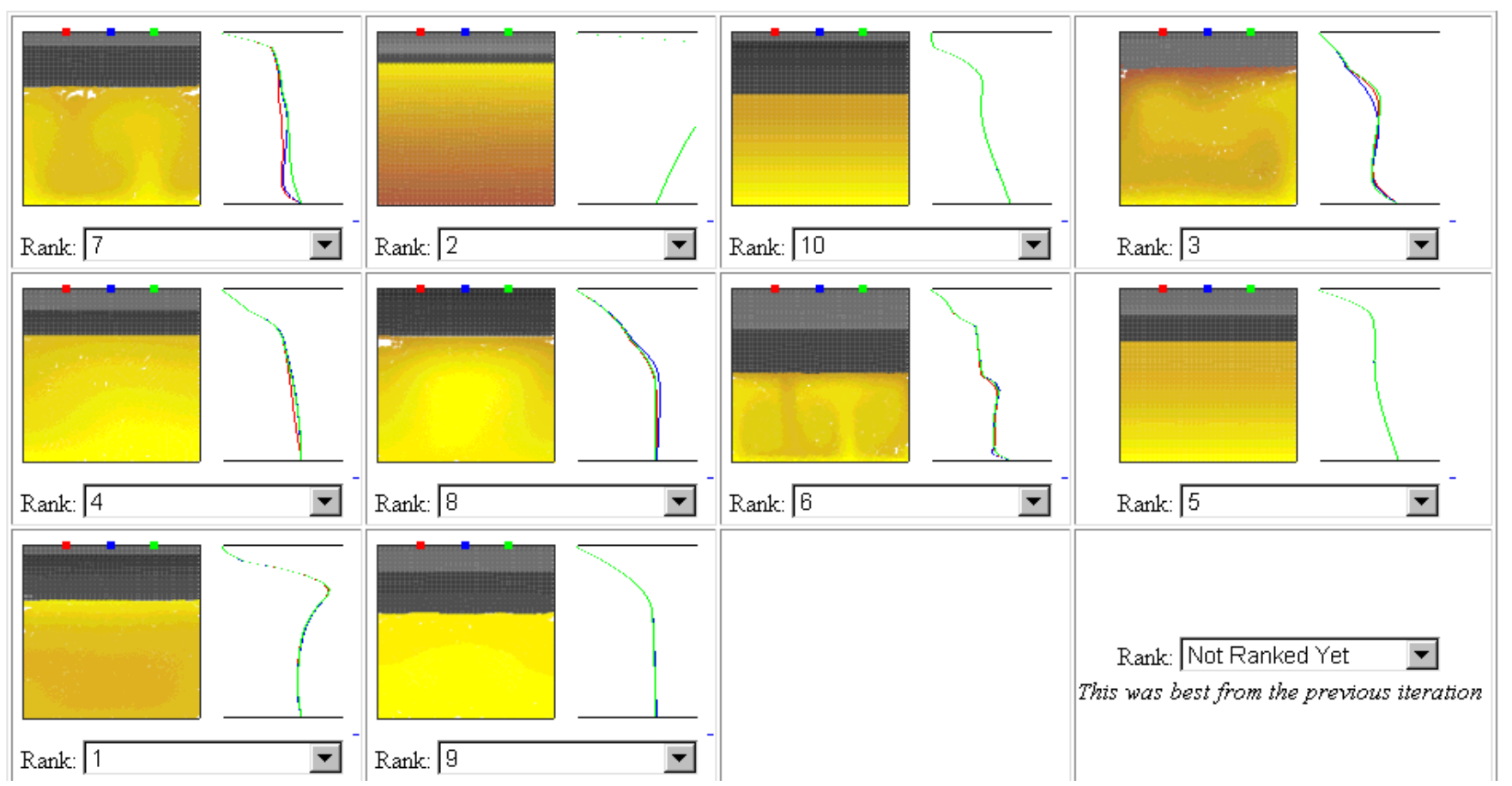

Figure 2. First generation of the interactive GA run. The sections are the final stages of animations initialized randomly.

In Figure 3 we see the result of the inversion at the third generation. Now together with the new 10 individuals, at the bottom right hand side we see the best individual from the previous generation. Keeping the best individual in a GA run is a standard operation. In this case it provides a way to rank the new generation according to previous results. The kink in the 'mantle' (dark hook), that is the typical feature of the target image starts to appear in the eighth individual. This animation will now be selected as best individual.

Figure 4 shows the eighth and last generation. As this is an exploratory test the choice of stopping at the eighth generation was purely by chance. In a real test we would have stopped as soon as a satisfactory match has been found. In this case we were interested in exploring the solution space and testing the GA behaviors. As we see many solutions "close" to the target have been found. The solutions found during this inversion process show a good fit to the modeled geotherm.

They also reproduce the structural features of the convection processes in the layer. These features are merely second order signatures of the physics of the system. However, such small clues which hint at how a process was initiated are vital in geology and it is reassuring to see that this information can be captured by the appropriate ranking choices.
The best results from the subjective inversion have eliminated one of the crustal layers. This highlights the fact that, for this simulation, some parameters produce first-order differences in the outcome, while others serve to fine-tune the result. The total crustal thickness has a first-order effect, but it was parameterized as a sum of two internal layers, whose relative thicknesses had a second order effect on the simulation. In the genetic algorithm, this is not an ideal situation because the relative thicknesses can not be constrained. The selection of parameters should be made more carefully. The first pass of the inversion does, however, serve as a way to analyse the parameter space to help ensure the parameters are chosen to be as independent as possible.

\subsection{Comparison with numerical inversion}

A second test was run on the same model. This consisted of a traditional numerical optimization. The misfit used was the squared error between the target temperature profile and the one generated as final result of the animation. The numerical inversion was run with exactly the same GA parameterization, number of individuals and number of generations. The result from both the interactive and numerical inversion can be seen in Figure 5. Both the quality of the result and the computational cost of the 2 inversions are comparable. 


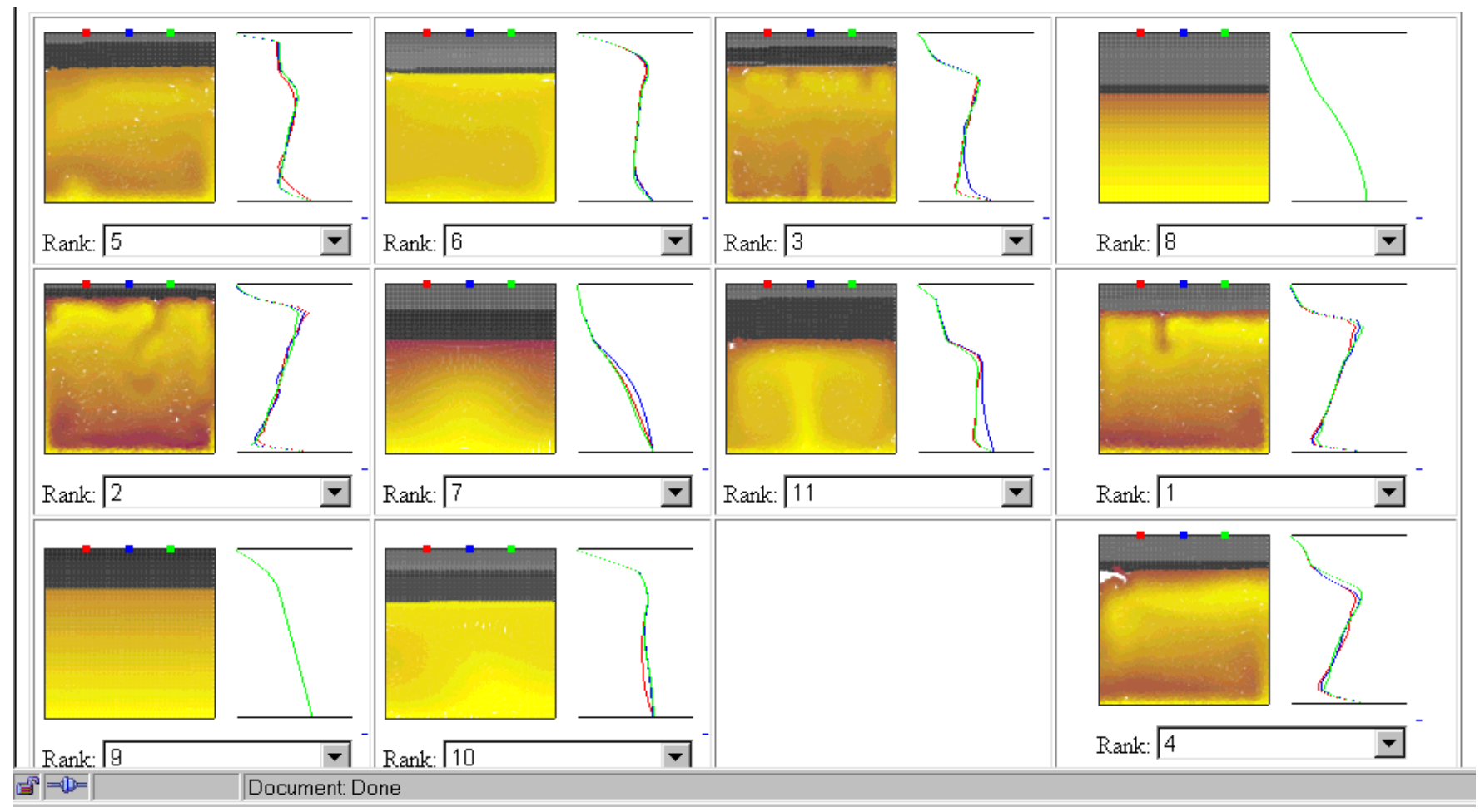

Figure 3. Third generation of the interactive GA run. The eighth individual starts to show strong similarities to the target section. Notice also that the best individual from the previous generation (eleventh individual) is used in the ranking stage.

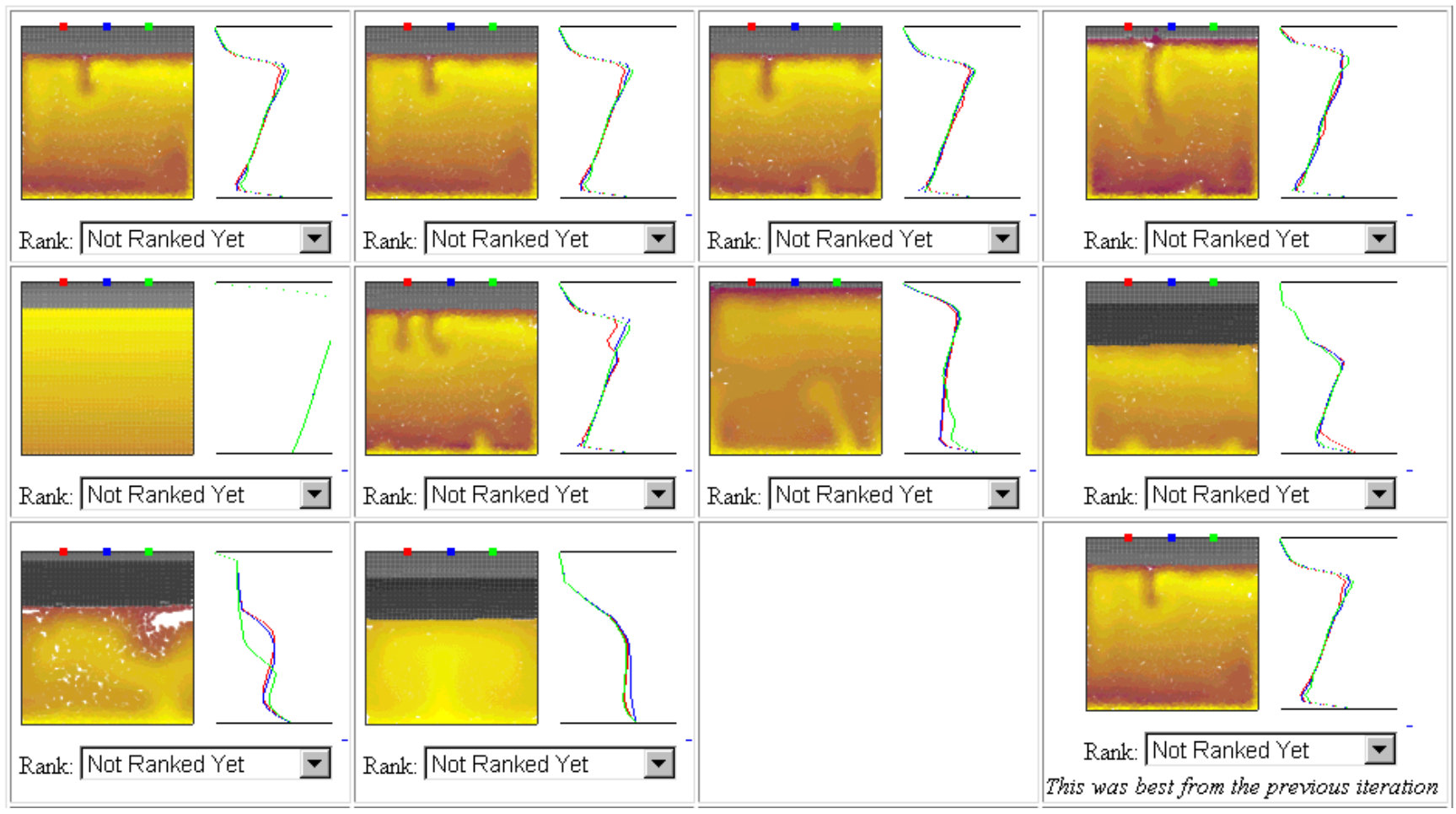

Figure 4. Last (eight) generation of the interactive GA run. Many individuals now show similarities to the target section. 

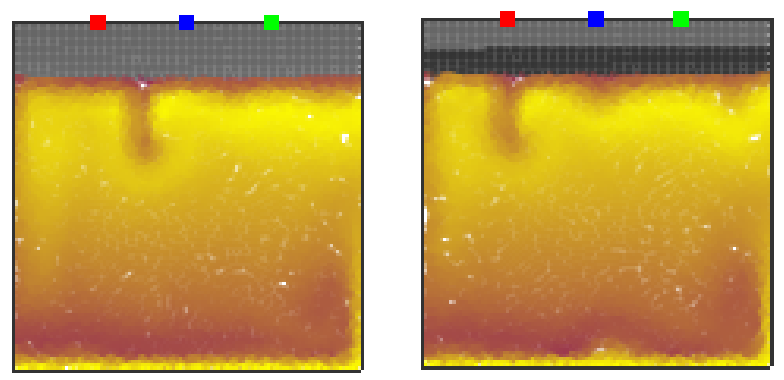

Figure 5. Best result from the interactive inversion (left) and numerical inversion (right)

A number of interesting conclusions can be drawn from this experiment:

1) The similarity between the numerical and human driven inversion is a very important result for geological applications, in which reliable data are rare and often sparse. For this specific application, as stated above, reliable temperature data can be obtained only close to the earth's surface, and measurements at depth can only be extrapolated from other data. In this test, the numerical inversion was given an unrealistic advantage in assuming error free temperature measurements along the entire profile. In real applications reliable temperature data at depth would be rare, inaccurate and at times absent. The ability of the geoscientist to direct the inversion to a successful solution without such data looks very promising.

2) During the human driven inversion, solutions characterized by specific features judged of particular relevance to the problem were selected even if their global similarity to the target image was relatively poor. Basically, the users had performed a sort of mental eigen-vector decomposition, with selection of what are considered the crucial directions of the search. This process is completely impossible in a traditional GA run, in which only data misfit, with no extra information is used.

3) The geoscientist was also using his/her knowledge of the inner mechanics of GA inversion in his/her choices, paying attention to leave certain 'good' features in the GA population even if belonging to low quality individuals. This is again impossible for a GA, that is 'unaware' of its own mechanics.

These last two strategies carry both advantages and disadvantages. The disadvantage lies in directing the GA run too much, with the risk of preventing its main feature, that is the global search. The advantages are the possibility of speeding up the search and using a priori information. Notice that in this case there would be a double use of a priori information, first in the subjective judgement, second in the ability to interfere with the standard GA run. Also, this provides the option of interactively controlling some GA parameters, like population size and rate of mutation, depending on the convergence speed and variability in the population. This offers a completely new avenue to explore.

4) While numerical inversion is sensitive only to the temperature profile, human driven inversion is sensitive particularly to geological structures and to dynamic evolution. Both are modeled as color images in the animation. Specific choices of the colors will allow the discrimination of certain features at the expenses of others. This confirms previous results on Interactive Inversions: the selection of a proper visualization and user interface becomes a crucial part of the inverse problem.

\section{Conclusions}

In scientific inverse problems measurements rarely supply sufficient constraints on a problem to allow for a unique and stable solution from inversion. Additional external constraints are used in these cases but are often constructed more for mathematical convenience than for strict physical appropriateness. This is because it is often hard to code analytically or numerically realistic a priori information. We have presented a simple way in which an inverse run can be driven entirely by subjective judgement from users with reasonable knowledge and experience. The method has proved to be successful and compared well to traditional numerical approaches. It requires only minimal time and effort from the user, most computational time being absorbed by computer forward modeling (as in any inverse application). We believe this technique can greatly widen the range of problems admissible for inversion and can be used for many scientific and industrial applications, either alone or in conjunction with traditional numerical techniques.

\section{Bibliography}

Kishi, K and Takagi, H. 1999, Developing montage systems for study on Interactive evolutionary computation: SOFT $3^{\text {rd }}$ workshop on Evaluation of Heart and Mind, 15-18, Gamagoori, Japan.

Takagi, H. 1998, Intercative evolutionary computation: system optimization based on human selective evaluation: IEEE Int. Conf. on Intelligent Engineering Systems, 1-6, Vienna, Austria, Sept 1998.

Takagi, H. 1998, Interactive evolutionary computation Cooperation of computational intelligence and human 
KANSEI: $5^{\text {th }}$ Int. Conf. on Soft Computing (IIZUKA 98) 41-

50, Iizuka, Japan. 